\title{
Multi-component Lattice Boltzmann simulation of the hydrodynamics in drip emitters
}

\author{
Giacomo Falcucci, ${ }^{1}$ Vesselin K. Krastev, ${ }^{2}$ Chiara Biscarini ${ }^{3}$ \\ ${ }^{1}$ Department of Enterprise Engineering, Tor Vergata University, Rome; ${ }^{2}$ Department of Economics and Management, \\ School of Engineering, University of Tuscia; ${ }^{3}$ UNESCO Chair in Water Resources Management and Culture, \\ University for Foreigners of Perugia, Italy
}

\begin{abstract}
In this paper, we propose a fast and efficient numerical technique based on the Lattice Boltzmann method (LBM) to model the flow through a reference drip emitter geometry. The aim of the study is to demonstrate the applicability of the LBM as a reliable simulation tool for the hydraulic optimisation of irrigation systems. Results for the water flow through a rectangular drip emitter are in good agreement with literature numerical and experimental data. Furthermore, we demonstrate the feasibility of the proposed model to simulate a multi-component flow that could be used to simulate the presence of additives, contaminants, and suspended particles.
\end{abstract}

\section{Introduction}

As shown in several studies, drip emitters play a major role in the hydraulic performance of drip irrigation systems (Glaad, 1974;

Correspondence: Chiara Biscarini, UNESCO Chair in Water Resources Management and Culture, University for Foreigners of Perugia, Piazza Fortebraccio 4, 06123 Perugia, Italy.

E-mail: chiara.biscarini@unistrapg.it

Key words: Drip emitters; Lattice Boltzmann; hydraulic modelling; multicomponent flows; clogging.

Acknowledgements and Funding: this work was supported by the Italian Ministry of Education, University and Research under PRIN grant No. 20154EHYW9 Combined numerical and experimental methodology for fluid structure interaction in free surface flows under impulsive loading. The numerical simulations were run on Zeus HPC facility, at the University of Naples Parthenope; Zeus was realised through the Italian Government Grant PAC01 00119 MITO Informazioni Multimediali per Oggetti Territoriali, with Prof. Elio Jannelli as the Scientific Responsible.

Received for publication: 11 December 2016.

Accepted for publication: 6 April 2017.

(C) Copyright G. Falcucci et al., 2017

Licensee PAGEPress, Italy

Journal of Agricultural Engineering 2017; XLVIII:649

doi:10.4081/jae.2017.649

This article is distributed under the terms of the Creative Commons Attribution Noncommercial License (by-nc 4.0) which permits any noncommercial use, distribution, and reproduction in any medium, provided the original author(s) and source are credited.
Ozekici et al., 1991; Carraro et al., 2006; Li et al., 2006). Glaad (1974) reports several results obtained under laboratory conditions and highlights the effect of structural form, dimension and material of emitter channels on the overall performance of the dripper. In Ozekici et al. (1991), pressure losses during water flow are related to the shape of the emitter channel.

However, a detailed experimental observation of the flow pattern through emitter channels has proven to be practically unfeasible, due to their complex shape and very small size (less than 1 $\mathrm{mm}^{2}$ of cross sectional area). This provides the typical framework in which computational fluid dynamics (CFD) can be proficiently employed (Xia and Sun, 2002; Lee, 2013; Lee et al., 2013), to allow an experimental-based optimisation of drip irrigation systems. To date, a few CFD applications to drip emitters can be reported in the scientific literature, all based on well established numerical techniques such as the finite volume method (FVM) or the finite element method (FEM) (Palau-Salvador et al., 2004; Wei et al., 2004, 2006; Provenzano et al., 2007; Li et al., 2008; Wu et al., 2013; Yurdem et al., 2015). In Palau-Salvador et al. (2004), a finite volume laminar flow model is employed to investigate the pressure $v s$ discharge rate correlation in a straight channel design, showing a good agreement with experimental data. In Wei et al. (2004) a novel channel design is simulated numerically, confirming that the use of CFD can effectively reduce the number of experimental samples and measurements. The FEM method is applied in Wei et al. (2006), under a turbulent flow assumption, to explore its reliability in the comparison of several labyrinth channel designs. Wu et al. (2013) analyse the effectiveness of the standard k- $\varepsilon$ model and the large eddy simulation (LES) method in simulating the fluid dynamics inside drip irrigation emitters and concluded that the LES model is more effective.

A potential candidate for the direct simulation (i.e., without turbulence modelling) of fluid flow in the micro-channels of drip emitters is the Lattice Boltzmann method (LBM), a powerful technique for the computational modelling of a wide variety of complex fluid flow problems (Benzi et al., 1992; Succi, 2001). The LBM philosophy consists into solving the macroscopic fluid dynamics through a mesoscopic (i.e., between micro and macro) kinetic approach, in which molecular details are neglected except those necessary to ensure mass, momentum and energy conservation at the macroscopic level. The method is then based on a very elegant and simple equation [Lattice Boltzmann equation (LBE)], which simulates transport phenomena through the evolution of density distribution functions (or populations).

Thanks to several improvements made in the last two decades, the LBM has revealed to be an accurate and efficient tool for modelling several complex fluid dynamics problems that could be of interest also for agricultural engineering, such as multiphase and multi-component flows, free-surface flows, flows in porous media, fluid-structure interaction, and micro-fluidics (Succi et al., 
1989; Ubertini et al., 2004; Falcucci et al., 2007; Colosqui et al., 2012; De Rosis et al., 2013; Falcucci et al., 2010, 2013; Zarghami et al., 2014a, 2014b; Chiappini et al., 2015; Di Francesco et al., 2015; Di Ilio et al., 2016, 2017; Krastev et al., 2016), including applications to complex geometries (Chiappini, 2015; Di Ilio et al., 2016; Krastev et al., 2016) and LBM extensions to non-regular and unstructured grids (Ubertini et al., 2004; Zarghami et al., 2014a, 2014b; Di Ilio et al., 2017).

The main advantage of LBM with respect to conventional CFD is represented by its simpler dynamics, which enables a straightforward and intrinsically parallel numerical implementation, and its second order accuracy without the need of a time marching procedure (Ubertini et al., 2010). Additionally, its molecular basis allows to easily incorporate the microscopic effects underlying macroscopic fluid phenomena, as opposed to numerical solvers derived from the Navier-Stokes equations.

In the present work LBM is applied for the first time, to the best of our knowledge, to the study the fluid dynamics of agricultural drip emitters. The paper is organised as follows: first, the numerical method is briefly explained, including the special procedure required for the definition of the computational domain; then, the method is applied to simulate a reference labyrinth channel pattern, comparing the results obtained with previous numerical and experimental findings; finally, results and future developments are briefly discussed.

\section{Numerical method}

In the Lattice Boltzmann approach the fluid flow is described by tracking the evolution of density distribution functions $f(\vec{x}, \vec{c}$, $t$ ) that describe the probability to find a system of particles at site $\vec{x}$, at time $t$ and with a velocity $\vec{c}$, discretized over a given stencil in $\vec{c}$ space. The general form of LBE reads as follows:

$$
f_{i}\left(\vec{x}+\vec{c}_{i} \Delta t, t+\Delta t\right)-f_{i}(\vec{x}, t)=\frac{f_{i}^{e q}(\vec{x}, t)-f_{i}(\vec{x}, t)}{\tau}
$$

in which the velocity space is reduced to few discrete points, $i=1$, $N$. Such a discretization derives from the assumption that at each site the particles can only move along a finite number of directions, $N$, described by a finite set of discrete particle velocity vectors, $\vec{c}_{i}$. In order to recover the correct fluid dynamic equations in the macroscopic limit, the set of discrete speeds is constructed to satisfy mass, momentum and energy conservation, and rotational symmetry. In this paper we refer to a 9 speeds discretization in 2 dimensions, called D2Q9.

The right hand side of Equation (1), called Bathnagar-GrossKrook (BGK) collision function Succi (2001), describes particles interaction through a single-time, $\tau$, relaxation toward the local equilibrium.

The local equilibrium fulfilling hydrodynamic conservation laws is described through the following second order polynomials in the velocity field:

$$
f_{i}^{e q}(\vec{x}, t)=\rho w_{i}\left[1+\beta \vec{c}_{i} \cdot \vec{u}+\frac{\left(\beta \vec{c}_{i} \cdot \vec{u}\right)^{2}}{2}-\beta|\vec{u}|^{2}\right]
$$

where $\beta=1 / c^{2}$, being $c_{s}$ the lattice sound speed, $\rho$ is the fluid density, $\vec{u}$ is the fluid speed and $w_{i}$ the associated weight coefficients.

The macroscopic quantities describing the fluid flow, such as density and velocity, are determined as hydrodynamic moments of the particle distribution functions:

$$
\rho(\vec{x}, t)=\sum_{i=1}^{N} f_{i}(\vec{x}, t)
$$

$\vec{u}(\vec{x}, t)=\frac{\sum_{i=1}^{N} c_{i} f_{i}(\vec{x}, t)}{\rho(\vec{x}, t)}$

The above formulation recovers incompressible hydrodynamics in the limit of weak departures from local equilibrium and near the incompressible limit (i.e., small Knudsen and Mach numbers), as shown through a Chapman-Enskog analysis in Succi (2001). Pressure and kinematic viscosity are calculated as follows:

$p(\vec{x}, t)=c_{s}^{2} \rho(\vec{x}, t)$

$v=c_{s}^{2}(\tau-\Delta t / 2)$

The LBM is computationally very efficient, as the streaming step moves explicitly the updated distribution functions to neighbouring nodes and the collision step is completely local, as it does not require any spatial or temporal derivatives. Moreover, thanks to the linear convection term and the linearized collision operator, the LBM can be easily parallelized. In several applications LBM has shown a clear superiority, in terms of computational performances, compared to FVM and FEM (Succi et al., 1989; Benzi et al., 1992; Succi, 2001; Falcucci et al., 2007, 2010, 2011; Colosqui et al., 2012; De Rosis et al., 2013, 2014; Falcucci et al., 2013; Di Francesco et al., 2015).

It is worth noting that the water flowing inside the drip emitters has also suspended particles inside, which may lead to channels clogging (Qingsong et al., 2008). As a first step towards particles modelling, our LBM model also incorporates multi-component transport, thus enabling the simultaneous description of species with different properties (e.g., different viscosity).

\section{Multi-component flow}

To account for the presence of different species, Eq. (1) can be rewritten in the following:

$$
f_{i}^{\alpha}\left(\vec{x}+\vec{c}_{i} \Delta t, t+\Delta t\right)-f_{i}^{\alpha}(\vec{x}, t)=\frac{f_{i}^{\alpha, e q}(\vec{x}, t)-f_{i}^{\alpha}(\vec{x}, t)}{\tau^{\alpha}}
$$

in which the index $\alpha$ spans on all the considered species: in our case, we have $\alpha=2$, corresponding to a carrier fluid and a passively transported second component.

In Eq. (7), the relaxation towards the local equilibrium of each species takes place on a distinct time scale, provided by $\tau^{\alpha}$.

The dynamical evolution of all the different species is coupled according to the equilibrium populations. Equation (2), in the multi-component formulation, is rewritten in the following form (Pfahler et al., 1990; Harley et al., 1995; Qisu and Xiaoyi, 1997; Kandilikar et al., 2003; Biscarini et al., 2013; Falcucci et al., 2016; Montessori et al., 2016):

$$
\begin{aligned}
& f_{i}^{1, e q}=\rho^{1} w_{i}\left[1+\beta \vec{c}_{i} \cdot \vec{u}+\frac{\left(\beta \vec{c}_{i} \cdot \vec{u}\right)^{2}}{2}-\beta|\vec{u}|^{2}\right], \text { for the carrier, } \\
& f_{i}^{\alpha, e q}=\rho^{\alpha} w_{i}\left[1+\beta \vec{c}_{i} \cdot \vec{u}\right], \text { for all the other species, }
\end{aligned}
$$

in which, $\vec{u}$ is the mean value of the macroscopic velocity, provided by:

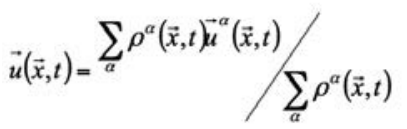


According to Eq. (7), it is possible to set different values for the species viscosities, in order to evaluate the behaviour of the passively advected species for different values of Peclét nondimensional number, defined as:

$$
\mathrm{Pe}=\frac{L U}{D}
$$

in which $L$ is a characteristic dimension and $U$ is the species macroscopic velocity magnitude.

According to the different values of species viscosity, different relaxation times $\tau^{\alpha}$ are retrieved, providing distinct behaviour of diffusion of Species 2 through the carrier, as reflected by the values of Peclét numbers of the transported species.

\section{Boundary conditions}

In the BGK scheme each fluid node receives information from the adjacent nodes (streaming step), but if one or more of the adjacent nodes belong to solid or open boundaries some populations of the fluid node are undetermined.

Most boundary conditions in the lattice Boltzmann method are based upon the so-called bounce-back scheme (Succi, 2001): when a particle collides with a stationary wall, it simply reverses its momentum. Therefore the particle distribution functions that stream from the fluid nodes to the wall nodes scatter back to the fluid node. The model presented in this paper uses the halfway wall bounce-back that can better simulate a no-slip or free-slip boundary by fictitiously placing the wall half way between a boundary site and an adjacent non-boundary site (Succi, 2001). Such a boundary treatment attains second-order accuracy Wei et al. (2004).

Bounce-back rules are also applied to the open boundaries, following the Zou and He approach (Qisu and Xiaoyi, 1997).

\section{Simulation setup}

In this section we report the results of the simulation of the hydrodynamics inside a rectangular drip emitter, whose 2D representation, together with its main geometrical dimensions, is shown in Figure 1.

The computational domain has been reconstructed starting from the details reported in Wei et al. (2006). We define the number of rectangular obstacles inside the $2 \mathrm{D}$ domain (i.e., 8 obstacles): such obstacles are created at fixed distances, then a free passage corresponding to $1 / 10$ of the computational domain length is left at the beginning and at the end of the computational domain, for stability purpose.

Simulations have been performed at different Reynolds numbers (Re), defined as follows:

$$
\operatorname{Re}=\frac{L U}{v}
$$

where $U$ is the average velocity within the channel and $L$ is the inlet height, which is chosen to be the characteristic length.

In the present simulations Re is varied between 50 and 250 by changing the relaxation parameter between 0.62 and 0.65 (in lattice units) and the characteristic length at the domain inlet. By changing the value of $\mathrm{L}$ in lattice units, we vary the scale of conversion between lengths in lattice units and in physical units, keeping the Reynolds similitude between the simulations and the real phenomenon. Finally, the water density has been assumed equal to $1000 \mathrm{~kg} / \mathrm{m}^{3}$.

At the upper limit of the considered Reynolds number interval, the flow through labyrinth-type channels could start exhibit a transitional behaviour (Pfahler et al., 1990; Harley et al., 1995; Kandilikar et al., 2003). As discussed above, some of the previous CFD studies on this matter are based on fully turbulent modelling assumptions, which is arguably an oversimplification of the actual flow physics.

Due to its inherent efficiency, LBM allows employing high fidelity computational grids (Biscarini et al., 2013), such that more flow details can be effectively captured. In this paper we employed different lattices for the different Reynolds number, as summarised in Table 1 which also reports the values of the Peclét number for the passively transported species.

\section{Results and discussion}

First we validate our approach by considering the mass conservation and evaluating the goodness of our modular reconstruction of the drip emitter.

Figure 2 shows the total mass evolution over time and shows that, after a first transient due to the initial conditions, the total mass reaches a constant value, as expected. This clearly indicates that mass conservation is ensured.

The performances of a drip emitter are usually measured through the relationship between the water flow rate and the pressure drop inside the labyrinth channels. Here we consider such a

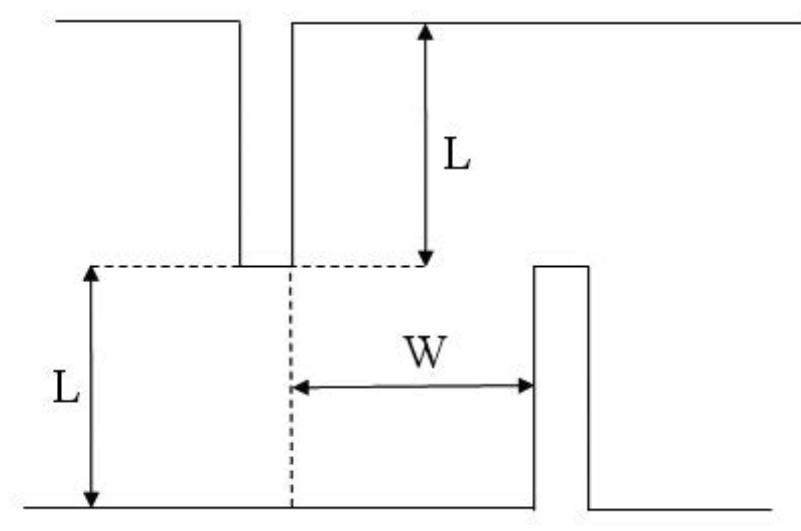

Figure 1. Schematic diagram of the labyrinth channels of a rectangular drip emitter. The width $w$ between the obstacles is retrieved from the total length of the emitter divided by the number of obstacles (i.e., 8).

Table 1. Lattice dimension for the different Reynolds and Peclét numbers.

\begin{tabular}{lcc} 
Lattice & Reynolds & Peclét \\
$800 \times 100$ & 50 & 50 \\
$960 \times 120$ & 150 & 150 \\
\hline $3600 \times 450$ & 250 & 250 \\
\hline
\end{tabular}


relationship by means of a non-dimensional pressure coefficient defined as follows:

$$
C_{p}=2 \frac{p_{\text {in }}-p_{\text {out }}}{\rho u^{2}}
$$

where $p_{\text {in }}$ and $p_{\text {out }}$ are the pressure values at the channel inlet and $\rho$ outlet, respectively, and $\rho$ is the density of the water at ambient conditions.

Figure 3 shows the variation of the pressure coefficient with the Reynolds number. Present numerical results are compared to experimental and numerical data available in Wei et al. (2006). The agreement with the experiments remains remarkably good in the whole range of the Reynolds number, including the highest considered Re values. Figure 4 shows the temporal evolution of a passively transported species with different levels of viscosity compared to the flow carrier (i.e., water). These results highlight the potential of the proposed method for the numerical modelling of drip emitters. The transported species, in fact, could be applied in several cases for drip emitters, as water for irrigation may contain additives, contaminants and suspended particles. For additives it is

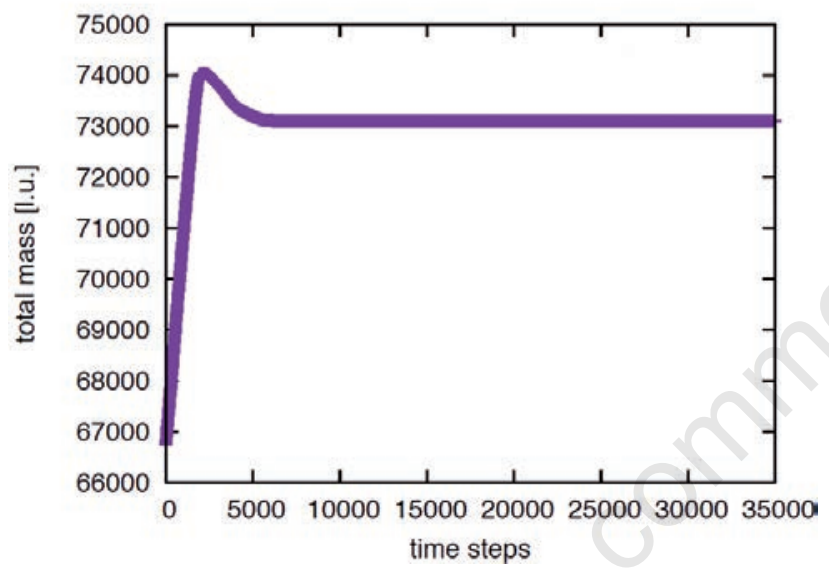

Figure 2. Check on mass conservation for the simulation corresponding to $R e=150$. l.u., lattice units.

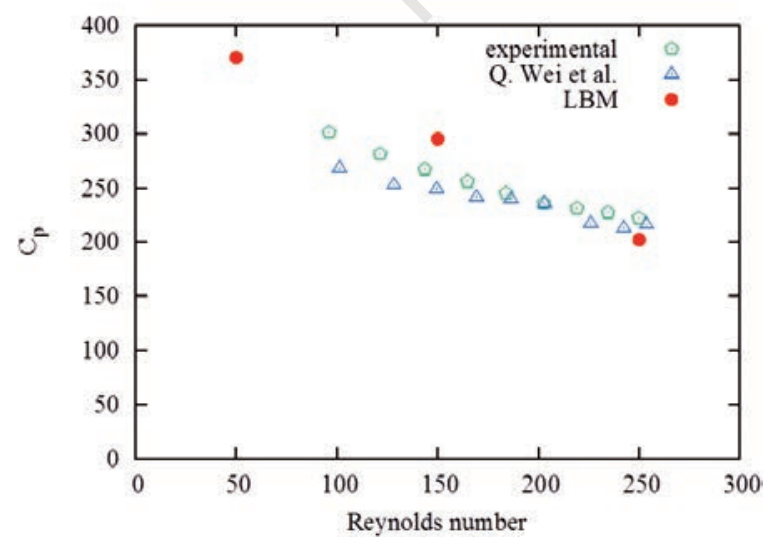

Figure 3. Comparison between the pressure coefficient and the Reynolds number. Our simulations are compared to the numerical and experimental results in Wei et al. (2004, 2006). LBM, Lattice Boltzmann method. crucial to understand how they transport inside the channel and if they reach homogeneously the channel exit. On the other hand, for suspended particles it is important to understand if they concentrate somewhere in the channel and cause channel clogging. Finally, contaminants may affect the irrigation or may damage the channels due to corrosion.

Results evidence how the species with the higher viscosity tends to spread out and accumulate behind the solid obstacles in the labyrinth channel, especially during the transient pass-through. Conversely, the low viscosity species tends to move across the channel in a smoother way, with less diffusion and local accumu-

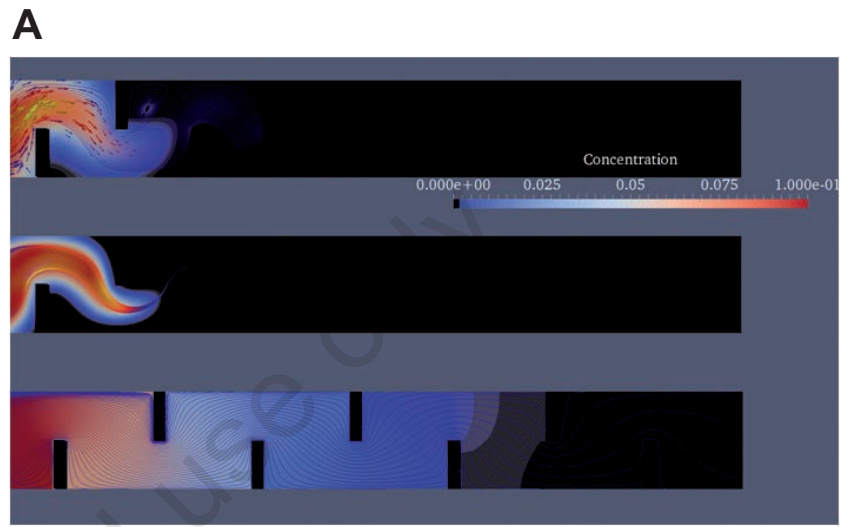

B

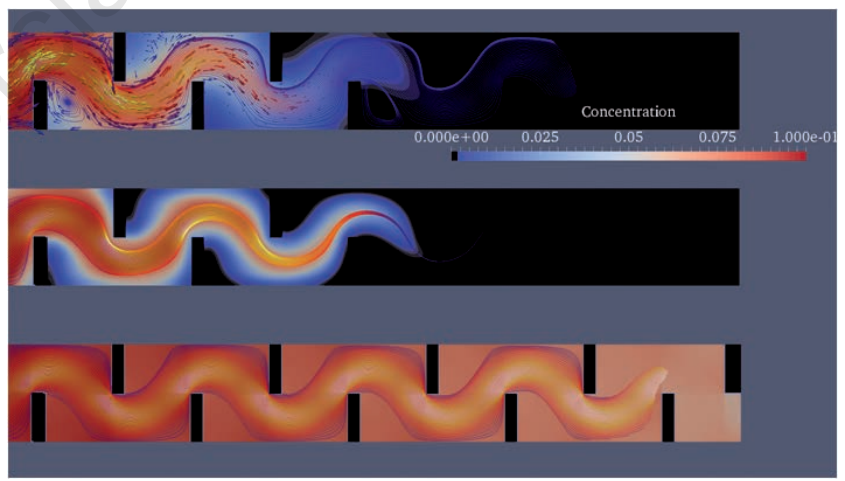

C

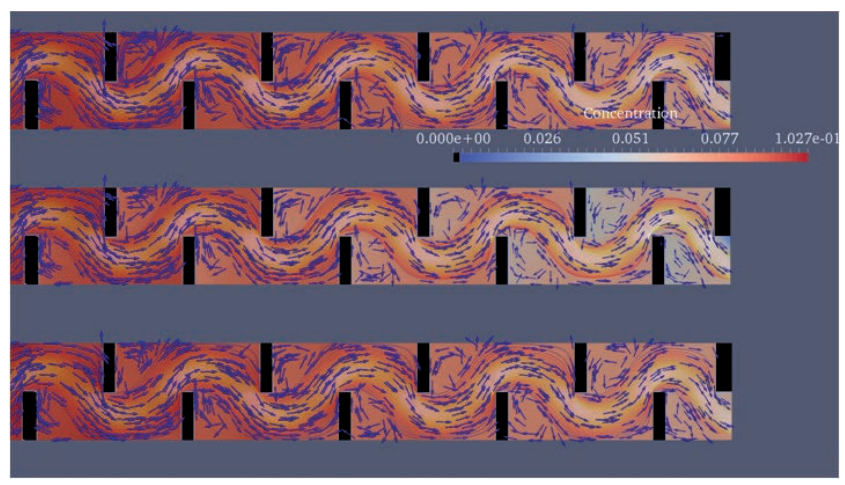

Figure 4. Density and velocity field of the passively transported species 2; A) $t=200$ time steps; B) $t=500$ time steps; C) at regime, $\mathbf{t}=20,000$ time steps. In each panel, we report from top to bottom the cases corresponding to: i) same viscosity between the species; ii) species 2 with higher viscosity; and iii) with lower viscosity. 
lation. This is in line with what expected, as the low viscosity species experiences a reduced drag in the interaction with the carrier and with the solid obstacles. This provides an interesting predictive tool for the development of new-generation agricultural sprinklers, designed in order to avoid the clogging for the accumulation of solid particles.

\section{Conclusions}

This work presents the first application of the Lattice Boltzmann method to the simulation of drip irrigation systems. In particular, we simulate the flow inside a rectangular labyrinth-type drip emitter channel at different Reynolds numbers. The numerical method revealed to be efficient and reliable. The comparison with literature data shows a good agreement between present and independent results, both numerical and experimental, for a Reynolds number ranging from 50 to 250 . Moreover, given that present simulations regard a multi-component flow, the model allows a detailed analysis of a passively transported specie, which could represent an additive, a contaminant or suspended nanoparticles. Future studies will include applications to different channel designs, as well as a further extension of the method to include suspended particle flow modelling.

\section{References}

Benzi R., Succi S., Vergassola M. 1992. The lattice Boltzmann equation: theory and applications. Phys. Rep. 222:145-97.

Biscarini C., Di Francesco S., Nardi F., Manciola P. 2013. Detailed simulation of complex hydraulic problems with macroscopic and mesoscopic mathematical methods. Math. Probl. Engine. 2013:928309.

Cararo D.C., Botrel T.A., Hills D.J., Leverenz H.L. 2006. Analysis of clogging in drip emitters during wastewater irrigation. Appl. Engine. Agricult. 22:251-7.

Chiappini D., Bella G., Festuccia A., Simoncini A. 2015. Direct numerical simulation of an open-cell metallic foam through Lattice Boltzmann method. Commun. Comput. Phys. 18:707-22.

Colosqui C.E., Falcucci G., Ubertini S., Succi S. 2012. Mesoscopic simulation of non-ideal fluids with self-tuning of the equation of state. Soft Matter. 8:3798-809.

De Rosis A., Falcucci G., Ubertini S., Ubertini F. 2013. A coupled lattice Boltzmann-finite element approach for two-dimensional fluid-structure interaction. Comput. Fluids. 86:558-68.

De Rosis A., Falcucci G., Ubertini S., Ubertini F. 2014. Aeroelastic study of flexible flapping wings by a coupled lattice Boltzmann-finite element approach with immersed boundary method. J. Fluids Struct. 49:516-33.

Di Francesco S., Biscarini C., Manciola P. 2015. Numerical simulation of water free-surface flows through a front-tracking lattice Boltzmann approach. J. Hydroinform. 17:1-6.

Di Ilio G., Chiappini D., Bella G. 2016. A comparison of numerical methods for non-Newtonian fluid flows in a sudden expansion. Int. J. Modern Phys. C. 27:1650139.

Di Ilio G., Chiappini D., Ubertini S., Bella G., Succi S. 2017. Hybrid lattice Boltzmann method on overlapping grids. Phys. Rev. E. 95:013309.

Falcucci G., Bella G., Chiatti G., Chibbaro S., Sbragaglia M., Succi S. 2007. Lattice Boltzmann models with mid-range interactions. Commun. Comput. Phys. 2:1071-84.
Falcucci G., Jannelli E., Ubertini S., Succi S. 2013. Direct numerical evidence of stress-induced cavitation. J. Fluid. Mech. 728:362-75.

Falcucci G., Succi S., Montessori A., Melchionna S., Prestininzi P., Barroo C., Bell D.C., Biener M.M., Biener J., Zugic B. Kaxiras E. 2016. Mapping reactive flow patterns in monolithic nanoporous catalysts. Microfluid. Nanofluid. 20:1-13.

Falcucci G., Ubertini S., Biscarini C., Di Francesco S., Chiappini D., Palpacelli S., De Maio A., Succi S. 2011. Lattice Boltzmann methods for multiphase flow simulations across scales. Commun. Comput. Phys. 9:269-96.

Falcucci G., Ubertini S., Succi S. 2010. Lattice Boltzmann simulations of phase-separating flows at large density ratios: the case of doubly-attractive pseudo-potentials. Soft Matt. 6:4357-65.

Glaad Y.K. 1974. Hydraulic and mechanical properties of drippers. In: Proceedings of the Second International Drip Irrigation Congress, University of California, Riverside, CA, USA.

Harley J.C., Huang Y.F., Bau H. 1995. Gas flow in micro channels. J. Fluid Mech. 284:257-74.

Kandilikar S.G., Joshi S., Tian S. 2003. Effect of surface roughness on heat transfer and fluid flow character at low Reynolds numbers in small diameter tubes. Heat. Transfer. Eng. 24:4-16.

Krastev V., Amati G., Jannelli E., Falcucci G. 2016. Direct numerical simulation of SCR reactors through kinetic approach. SAE Technical Paper 2016-01-0963. [Epub ahead of print].

Lee I.B. 2013. Trends in CFD applications in agriculture. Acta Hortic. 1008:19-26.

Lee I.B., Bitog J.P.P., Hong S.W., Seo I.H., Kwon K.S., Bartzanas T., Kacira M. 2013. The past, present and future of CFD for agro-environmental applications. Comput. Electron. Agricult. 93:168-83.

Li Y.K., Yang P.L., Ren S.M., Xu T.W. 2006. Hydraulic characterizations of tortuous flow in path drip irrigation emitter. J. Hydrodyn. B. 18:449-57.

Li Y., Yang P., Xu T., Ren S., Lin X., Wei R., Xu H. 2008. CFD and digital particle tracking to assess flow characteristics in the labyrinth flow path of a drip irrigation emitter. Irrig. Sci. 26:427-38.

Montessori A., Prestininzi P., La Rocca M., Falcucci G., Succi S., Kaxiras E. 2016. Effects of Knudsen diffusivity on the effective reactivity of nanoporous catalyst media. J. Comput. Sci. 17:377-83.

Ozekici B., Sneed R.E., Ronald E. 1991. Analysis of pressure losses in tortuous path emitters. ASAE Paper No. 912155. ASAE, St. Joseph, MI, USA.

Palau-Salvador G., Arviza-Valverde J., Bralts V.F. 2004. Hydraulic flow behaviour through an in-line emitter labyrinth using CFD techniques. ASAE Paper No. 042252. ASAE, St. Joseph, MI, USA.

Pfahler J.N., Harley J., Bau H. 1990. Liquid and gas transport in small channels. ASME DSC 19:149-57.

Provenzano G., Dio P.D., Salvador G.P. 2007. New computational fluid dynamic procedure to estimate friction and local losses in coextruded drip laterals. J. Irrig. Drain. Engine. 133:520-7.

Qingsong W., Gang L., Jie L., Yusheng S., Wenchu D., Shuhuai, H. 2008. Evaluations of emitter clogging in drip irrigation by twophase flow simulations and laboratory experiments. Comput. Electron. Agricult. 63:294-303.

Qisu Z., Xiaoyi H. 1997. On pressure and velocity boundary conditions for the lattice Boltzmann BGK model. Phys. Fluids. 9:1592-8.

Succi S. 2001. The Lattice Boltzmann equation: for fluid dynamics and beyond. Oxford University Press, Oxford, UK.

Succi S., Foti E., Higuera F. 1989. Three-dimensional flows in 
complex geometries with the lattice Boltzmann method. EPL Europhys. Lett. 10:433.

Ubertini S., Asinari P., Succi, S. 2010. Three ways to lattice Boltzmann: a unified time-marching picture. Phys. Rev. E. 81:016311.

Ubertini S., Succi S., Bella G. 2004. Lattice Boltzmann schemes without coordinates. Phil. Trans. R. Soc. Lond. A. 362:1763-71.

Wei Q.S., Shi Y.S., Dong W.C., Lu G., Huang S.H. 2006. Study on hydraulic performance of drip emitters by computational fluid dynamics. Agric. Water Manage. 84:130-6.

Wei Q.S., Shi Y.S., Lu J., Dong W.C., Huang S.H. 2004. Study on theory and process to rapidly develop drip emitters with low cost. Trans. Chin. Soc. Agric. Eng. 21:17-21.

Wu D., Li Y.K., Liu H.S., Yang P.L., Sun H.S., Liu Y.Z. 2013. Simulation of the flow characteristics of a drip irrigation emitter with large eddy methods. Math. Comput. Model. 58:497-506.

Xia B., Sun D. W. 2002. Applications of computational fluid dynamics (CFD) in the food industry: a review. Comput. Electron. Agric. 34:5-24.

Yurdem H., Demir V., Mancuhan A. 2015. Development of a simplified model for predicting the optimum lengths of drip irrigation laterals with coextruded cylindrical in-line emitters. Biosyst. Engine. 137:22-35.

Zarghami A., Biscarini C., Succi S., Ubertini S. 2014a. Hydrodynamics in porous media: A finite volume lattice Boltzmann study. J. Sci. Comput. 59:80-103.

Zarghami A., Francesco S. D., Biscarini C. 2014b. Porous substrate effects on thermal flows through a rev-scale finite volume lattice Boltzmann model. Int. J. Modern Phys. C. 25:1350086. 